\title{
All-Optical Transistor Action in Photonic Band Gap Materials
}

\author{
Marian Florescu and Sajeev John \\ Department of Physics, University of Toronto, Toronto, Ontario, Canada M5S 1A7
}

\begin{abstract}
We describe all-optical transistor action in photonic band gap materials doped with active atoms. In the presence of a photonic band gap (PBG) material, a coherent laser beam with the frequency slightly detuned from the resonant atomic transition frequency can drive a collection of two-level atoms to an almost totally inverted state, a phenomenon strictly forbidden in ordinary vacuum. By changing the laser field intensity in the neighborhood of a threshold value, it is possible to drive the atomic system through a transition from states in which the atoms populate preferentially the ground level to almost totally inverted states. In this process, the atomic system switches from a passive medium (highly absorptive) to a active medium (highly amplifying). The switching action in a PBG material is not associated with operating near a narrow cavity resonance with conventional trade-off between switching time and switching threshold intensity. Rather it is associated with an abrupt discontinuity in the engineered broad band electromagnetic density of states of the PBG material. We demonstrate all-optical transistor action in PBG materials by analyzing the absorption spectrum of a second probe laser beam and we show that the probe beam experience a substantial differential gain by slight intensity modulations in the control laser field.
\end{abstract}

Keywords: All-optical transistor, all-optical switching, photonic band gap materials, collective atomic switching, resonance fluorescence.

\section{INTRODUCTION}

The transmission of information as optical signals encoded on light waves traveling through optical fibers and optical networks is increasingly moving to shorter and shorter distance scales. In the near future, optical networking is poised to supersede conventional transmission over electric wires and electronic networks for computer-to-computer communications, chip-to-chip communications, and even on-chip communications. The ever-increasing demand for faster and more reliable devices to process the optical signals offers new opportunities in developing all-optical signal processing systems. All-optical switches, two-state and many-state all-optical memories, all-optical limiters, all-optical discriminators and all-optical transistors ${ }^{1}$ are only a few of the many devices proposed during the last two decades. In particular, it has been suggested that electronic transistors may one day be replaced by all-optical transistors, which will perform all the operations that their electronic counterparts do: amplification, switching, modulation and detection. The "all-optical" label is commonly used to distinguish the devices do not involve dissipative electronic transport ${ }^{2}$ and require essentially no electrical communication of information. The all-optical transistor action was first observed in the context of optical bistability $^{1,3}$ and consists in a strong differential gain regime, in which, for small variations in the input intensity, the output intensity has a very strong variation. This analog operation is for all-optical input what transistor action is for electrical inputs. The emerging competition between all-optical devices and their electronic counterparts brings with it significant practical challenges. The most important demand is related to the integration of the all-optical devices. In order to achieve an all-optical chip, the individual components should have length scales of the ten-micrometer order. In order to diminish the heating of the chip and the operating power, the holding power and the switching energy should be minimized. The large number of interconnections on an all-optical chip should be insensitive to environmental perturbation and should operate at room temperature.

Further author information: (Send correspondence to M.F.)

M.F.: E-mail: marian@physics.utoronto.ca 


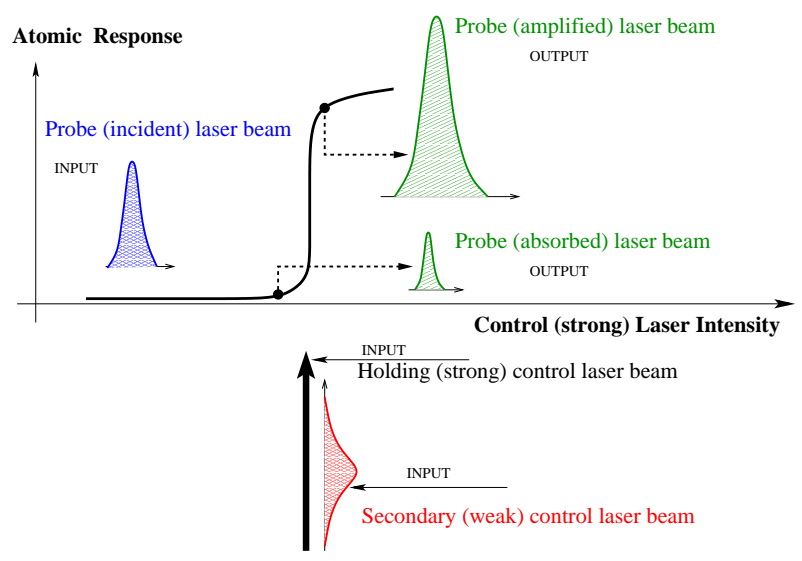

Figure 1. Schematic description of the transistor action. ${ }^{1,4}$ The system response exhibits a strong variation at a well defined threshold value of the control laser field intensity. A second weak control laser field (whose magnitude is determined by the width of the threshold region between absorbing and amplifying states) is used to switch the device. An incident probe beam will be absorbed or amplified depending upon the magnitude of the total control laser field.

\section{LOW THRESHOLD ALL-OPTICAL SWITCHING AND TRANSISTOR ACTION NEAR A PHOTONIC BAND GAP}

Photonic band gap materials constitute a new class of dielectrics, which carry the concept of moulding and controlling the flow of light to its most microscopic level. This is entailed in a fundamentally new optical principle, namely the localization of light ${ }^{5,6}$ and leads to the inhibition of spontaneous emission, ${ }^{7}$ the formation of the photon-atom bound states, ${ }^{8}$ very low threshold nonlinear optical phenomena ${ }^{9}$ and low threshold collective atomic switching behavior. In the context of all-optical transistor action, the photonic band edge, which separates the PBG from the continuum of propagating electromagnetic modes, facilitates fundamental switching effects for resonant two-level systems that are forbidden in ordinary vacuum. In particular, the Einstein rate equations, ${ }^{10}$ when applied to a collection of two-level atoms coherently pumped near resonance in ordinary vacuum, forbid atomic population inversion in the steady state limit. Such inversion often plays a key role in the laser light emission. As a consequence, it is customary to pump an active medium to a higher level, followed by incoherent relaxation to the (population inverted) excited level that defines the lasing transition. This type of switching from the ground to the excited state involves incoherent processes and makes the conventional laser unsuitable as an ultra-fast optical switch or transistor. Moreover, the pumping threshold required to achieve inversion may be unsuitable for all-optical information processing. On the other hand, in a photonic crystal, a coherent laser beam with the frequency slightly detuned from the atomic transition frequency can drive a two-level atom to almost totally inverted atomic states. ${ }^{4,9,11}$ In this process, the atomic system switches from a passive medium (highly absorptive) to a gain medium (highly amplifying), as a function of the external driving field. The large differential gain exhibited by the atomic medium is very robust with respect to non-radiative relaxation and dephasing mechanisms. ${ }^{11}$ This switching effect exhibits collective enhancement when there are many two-level atoms within a cubic wavelength inside the photonic band gap material. ${ }^{4,9}$

A doped PBG material may, in this sense, be used as an ultra-fast all-optical switch and an all-optical micro-transistor. Similar to other all-optical transistor proposals, ${ }^{1}$ the transition between the absorptive and the amplifying regime can be modulated with a weak second control laser field (in phase and in resonance with the main strong pump field). Unlike other proposals, the switching effect is not limited to the coupling of light to a narrow cavity resonance in which there is a conventional (inverse) relationship between the switching (cavity build-up) time and the switching intensity threshold. Instead, the PBG based switching effect requires the coupling of light emitters to a broad band electromagnetic density of modes in which there is a sharp (discontinuous) jump over a narrow frequency interval. The engineering of the defect structure within a 3D PBG provides considerable latitude in the development of such a device heterostructure. As we show in Section 4, 


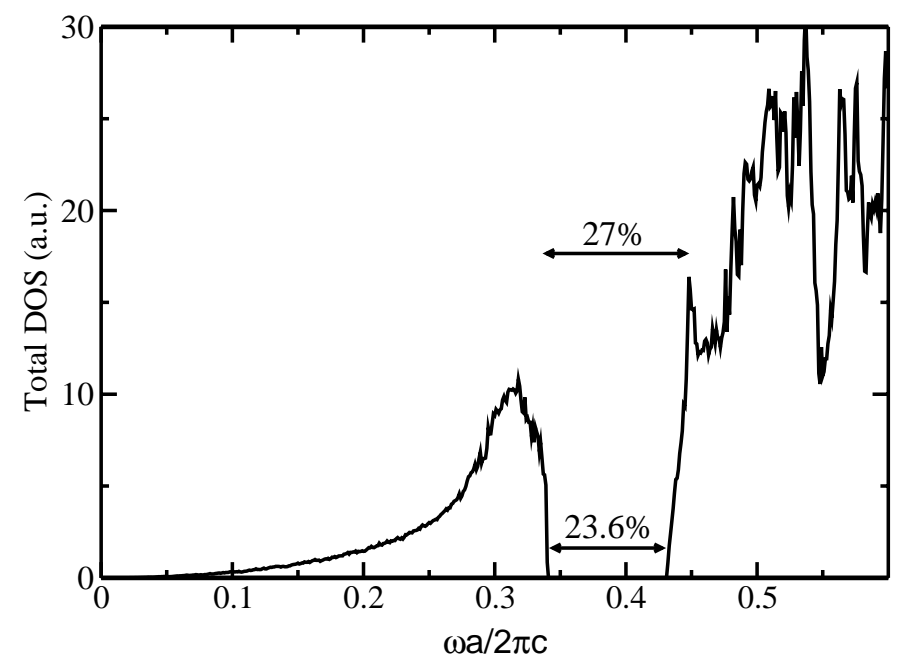

Figure 2. The total electromagnetic density of states for an optimized inverted square spirals structure. ${ }^{23}$ Here $\omega$ is the frequency of the light, $a$ is the lattice constant of the dielectric structure, and $c$ the speed of light.

a probe laser field of prescribed wavelength will experience a substantial differential gain by slight modulations in the weak control laser field. Under certain conditions, the fluctuations in the number of the excited atoms that contribute to the amplification process can be strongly diminished (the statistics of the excited atoms becomes sub-Poissonian ${ }^{9}$ ), which in turn determine a low noise regime of amplification. ${ }^{4}$

\subsection{Optical Mode Density near a Photonic Band Gap}

PBG materials typically consist of a two inter-penetrating dielectric components. The first is a connected high dielectric constant backbone, and the second is a connected low dielectric constant network. For example, a network of criss-crossing pores in a semiconductor of refractive index $3.5,{ }^{12}$ or a woodpile structure ${ }^{13,14}$ can provide band gaps of $20 \%$ of the central frequency of the gap. A detailed review of recent advances in the fabrication of 3D photonic band gap materials is given in Ref.15. A fabrication method using photoelectrochemical etching pores has been also proposed in Refs. 16,17. A recent breakthrough using self-assembly methods, ${ }^{18-21}$ is a silicon based inverse opal 3D photonic crystal with a $5 \%$ complete $3 \mathrm{D}$ photonic band gap centered near $1.5 \mu \mathrm{m} .{ }^{22}$ Very recently, ${ }^{23}$ based on glancing angle deposition methods, a blueprint for a 3D photonic band gap material amenable to large-scale micro fabrication on the optical scale has been suggested. The proposed chiral crystal consists of square spiral posts on a tetragonal lattice, and for silicon posts in air background (direct structure) exhibits a full band gap of $15 \%$ of the central frequency, while the inverse structure (air posts in a silicon background), the band gap can be as large as $24 \%$. The photon density of states for this inverse square spiral is shown in Figure 2.

In a real 3D photonic band gap material, the electromagnetic modes near the upper photonic band edge are concentrated in the air fraction of the composite material, and accordingly, this is referred to as the air band. Near the lower photonic band edge, the electromagnetic energy is concentrated in the high dielectric backbone. Accordingly, this is referred to as the dielectric band. The active "two-level atoms" in our model calculation can be embedded as quantum dots in the dielectric backbone or may be laser cooled and trapped into the void regions of the PBG material. ${ }^{24}$ As can be seen from Figure 2, if the two level atomic resonance occurs near a photonic band edge, the density of electromagnetic modes available to the atomic transition varies very rapidly with frequency. Inside the gap this density vanishes, whereas just outside the gap, the density 




Figure 3. Possible device heterostructure for all-optical switching and transistor action. From the left, two low-intensity laser beams enter the heterostructure from optical fibers combined by a coupler. The heterostructure consists of a planar waveguide region, containing quantum dots, sandwiched by a 3D PBG. From above, the heterostructure is illuminated by the main control (pump) laser field. On right of the figure, is seen the amplified signal field as it leaves the heterostructure.

may exceed that of ordinary vacuum. The ability of an external pump laser to switch spectral characteristics of the atomic system across the band edge (or near any other discontinuous density of states profile) leads to coherent all-optical switching and transistor action. In Figure 3, we show an artist view of an all-optical transistor based on a 3D PBG. The heterostructure consists of a 3D PBG template into which a sequence of III-V semiconductors has been infiltrated. The middle layer, which acts as a planar waveguide inside a 3D PBG, is an active region (quantum dots) sandwiched in between semiconductor cladding layers. The active region is assumed to experience a band edge type discontinuity within the larger 3D PBG. The laser beam from above the structure constitutes the main control (pump) laser field, which drives the "atomic" system embedded in the square spiral heterostructure. From the left, two low-intensity laser beams enter the heterostructure from optical fibers combined by a coupler, as shown on the left of the figure. One of them adds to the main pump beam with the role of switching the active medium between the absorptive and amplifying regimes. The second beam constitutes the signal field, which is amplified or absorbed (depending on the magnitude of the modulated component of the pump field) as it propagates through the active medium.

\section{SINGLE ATOM SWITCHING IN PHOTONIC BAND GAP MATERIALS}

We begin by reviewing the conventional picture of atom-field interaction, in which a coherent laser field with an average incident energy density $W$ and frequency $\omega$, interacts with a collection of $N$ independent two-level atoms in ordinary vacuum. In the most simplified picture, the dynamical and steady state behavior of the atomic system is governed by the Einstein rate equations, obtained under the assumption that the density of states characterizing the photonic reservoir is slowly varying with the frequency. The rate equation (based on Fermi's Golden rule for absorption and emission) is

$$
\frac{d N_{2}}{d t}=-\left(A+B_{a b} W\right) N_{2}+B_{e m} W N_{1},
$$

where $N_{2}$ is the number of excited atoms, $N_{1}$ is the number of unexcited atoms and $N=N_{1}+N_{2}$ is the total number of atoms. $A, B_{e m}, B_{a b}$ are spontaneous emission rate, the stimulated emission rate and the absorption rate, respectively. In ordinary vacuum, the stimulated emission rate and the absorption rate are equal $B_{a b}=B_{a m}=B$, so that in steady-state conditions the ratio of the number of excited atoms with respect to the total number of atoms is given by

$$
\frac{N_{2}}{N}=\frac{1}{2+A /(B \cdot W)}
$$


Clearly, the magnitude of the excited atomic population per atom $N_{2} / N$, has an upper bound of $\frac{1}{2}$, and in the case of ordinary vacuum, implicitly, prohibits positive values of the atomic inversion. As a result, coherent optical switching is not possible in this manner in ordinary vacuum. However, near a photonic band edge (or other sharp feature in the density of states) the underlying basis for Eq. (1) is no longer valid. Fermi's Golden rule for absorption and emission of light from the atom requires that the density of states in the electromagnetic reservoir is smooth and featureless. In a more detailed picture, the interaction of the external laser with the atom leads to a set of "dressed atomic states" 25 which are displaced in frequency relative to the "bare" atomic levels. Equation (1) is no longer appropriate if the density of states exhibits significant variation on the frequency scale of the dressed atomic level shift. In fact we show in the following analysis that population inversion and other unusual effects can be achieved at relatively low coherent pumping thresholds, if the density of states "discontinuity" is sufficiently large.

\subsection{Model Hamiltonian}

The rapid variations in the photonic density of states (DOS) with frequency in a PBG material lead to fundamental modifications in the response of a two-level system to an external laser field relative to ordinary vacuum. In this section we consider an effective mass approximation to the full dispersion relation of a photonic crystal. For a real dielectric crystal with an allowed point-group symmetry, the band edge occurs at certain points along the Bragg planes of the lattice and the electromagnetic dispersion relation may be approximated by $\omega(\mathbf{k})=\omega_{C}+A\left(\mathbf{k}-\mathbf{k}_{0}\right)^{2}$, where $\mathbf{k}_{0}$ is a point of the Brillouin zone boundary associated with the band edge. We consider a two-level atom interacting with a quantized electromagnetic field of a photonic crystal, and driven by an external single mode laser field. In the rotating wave approximation (RWA) and in a frame of reference rotating with the atomic resonance frequency, $\omega_{L}$, the Hamiltonian describing the total system is. ${ }^{11}$

$$
\begin{aligned}
H & =H_{0}+H_{1}, \\
H_{0} & =\sum_{\lambda} \hbar \Delta_{\lambda} a_{\lambda}^{\dagger} a_{\lambda}+\frac{1}{2} \hbar \Delta_{A L} \sigma_{3}+\hbar \varepsilon\left(\sigma_{12}+\sigma_{21}\right), \\
H_{1} & =i \hbar \sum_{\lambda} g_{\lambda}\left(a_{\lambda}^{\dagger} \sigma_{12}-a_{\lambda} \sigma_{21}\right),
\end{aligned}
$$

with

$$
g_{\lambda}=\frac{\omega_{A} d_{21}}{\hbar}\left[\frac{\hbar}{2 \epsilon_{0} \omega_{\lambda} \mathcal{V}}\right]^{1 / 2} \mathbf{e}_{\lambda} \cdot \mathbf{u}_{d}
$$

and $\sigma_{i j}=|i\rangle\langle j|(i, j=1,2)$ are the atomic pseudo-spin operators, $\sigma_{3}=\sigma_{22}-\sigma_{11}$ describes the atomic inversion, $a_{\lambda}$ and $a_{\lambda}^{\dagger}$ are the radiation field annihilation and creation operators, and $\Delta_{\lambda}=\omega_{\lambda}-\omega_{L}, \Delta_{A L}=\omega_{A}-\omega_{L}$ represents the $\lambda$-mode (atomic system) detuning frequency from the atomic frequency. Here, the atomic dipole moment $\mathbf{d}$ has been chosen real without loss of generality by a convenient choice of the atomic Hamiltonian eigenstates $|1\rangle,|2\rangle$ phases, with $d_{21}$ its magnitude and $\mathbf{u}_{d}$ the unit vector. $\mathbf{e}_{\lambda} \equiv \mathbf{e}_{\mathbf{k}, \sigma}$, with $\sigma=1,2$, are the transverse polarization vectors of the radiation field, and $\mathcal{V}$ is the quantization volume. The driving field is characterized by the Rabi frequency, $\varepsilon=\mathbf{d}_{21} \cdot \mathcal{E} / \hbar$, with $|\mathcal{E}|$ the laser electric field magnitude.

Furthermore, we diagonalize the atom plus external field part of the total Hamiltonian. This is done by a unitary transformation to the dressed atomic basis, defined by $|\tilde{1}\rangle=c|1\rangle+s|2\rangle,|\tilde{2}\rangle=-s|1\rangle+c|2\rangle$, where $c=\cos \phi, s=\sin \phi, \sin \phi^{2}=\frac{1}{2}\left[1-\operatorname{sign}\left(\Delta_{A L}\right) /\left(4 \epsilon^{2} / \Delta_{A L}^{2}+1\right)^{1 / 2}\right]$ and $0 \leq \phi \leq \pi / 2$. The corresponding dressed atomic operators, $R_{i j}=|\tilde{i}\rangle\langle\tilde{j}|(i, j=1,2), R_{3}=R_{22}-R_{11}$, are related to the bare atomic operators by

$$
\left\{\begin{array}{l}
\sigma_{12}=c s R_{3}+c^{2} R_{12}-s^{2} R_{21} \\
\sigma_{21}=c s R_{3}-s^{2} R_{12}+c^{2} R_{21} \\
\sigma_{3}=\left(c^{2}-s^{2}\right) R_{3}-2 c s\left(R_{12}+R_{21}\right)
\end{array} .\right.
$$

This transformation leads to the non-interacting dressed state Hamiltonian

$$
H_{0}=\hbar \Omega R_{3}+\hbar \sum_{\lambda} \Delta_{\lambda} a_{\lambda}^{\dagger} a_{\lambda}
$$


with $\Omega=\left[\epsilon^{2}+\Delta_{A L}^{2} / 4\right]^{1 / 2}$, the generalized Rabi frequency. The dressed atomic operators (in the interaction picture) exhibit the time dependence given by $\tilde{R}_{12}(t)=R_{12}(0) \exp (-2 i \Omega t), \quad \tilde{R}_{21}(t)=R_{21}(0) \exp (2 i \Omega t)$, and $\tilde{R}_{3}(t)=R_{3}(0)$. Clearly, $\tilde{R}_{3}(t), \tilde{R}_{12}(t), \tilde{R}_{21}(t)$ can be considered as source operators for the central component, left and right side-bands of the Mollow triplet at the frequencies $\omega_{L}, \omega_{L}-2 \Omega, \omega_{L}+2 \Omega$.

In this analysis, we use a perturbative approach to describe the first-order non-Markovian corrections to the resonance fluorescence phenomena in PBG materials. The perturbation parameter is the coupling constant between the atomic system and the photonic reservoir associated with the dielectric structure. The magnitude of the this coupling is directly related to the photonic DOS in the neighborhood of the resonant atomic frequency $\omega_{A}$. For the anisotropic model used in this section, the photonic DOS is continuous and finite over the entire spectral range of interest (in the effective mass approximation, the DOS of an anisotropic PBG material is given by

$$
\rho(\omega) \propto\left\{\begin{array}{cll}
\left(\omega-\omega_{C}\right)^{1 / 2} & \text { if } \quad \omega \geq \omega_{C} \\
0 & \text { if } \quad \omega<\omega_{C}
\end{array}\right.
$$

) and, remarkably, the spectral region surrounding the band-edge frequency $\omega_{C}$, which is associated with the most prominent non-Markovian effects, it is characterized by a relatively low magnitude of the density of states and, implicitly, the dynamics of the atomic system can be analyzed within of the framework of a perturbation approach.

We introduce the memory functions $G_{0}\left(t-t^{\prime}\right)=\sum_{\lambda} g_{\lambda}^{2} e^{-i \Delta_{\lambda}\left(t-t^{\prime}\right)}$ and $G_{ \pm}\left(t-t^{\prime}\right)=\sum_{\lambda} g_{\lambda}^{2} e^{-i\left(\Delta_{\lambda} \pm 2 \Omega\right)\left(t-t^{\prime}\right)}$. In general, the memory functions $G_{0}\left(t-t^{\prime}\right)$ and $G_{ \pm}\left(t-t^{\prime}\right)$ are determined by the radiation field density of states. For a broad band, smoothly varying density of states of the photonic reservoir (as in ordinary vacuum), the dependence of the memory functions on the external field can be ignored and the memory time associated with the photonic reservoir can be considered negligible small (the Markovian approximation) and simplify $G_{0}\left(t-t^{\prime}\right)=G_{ \pm}\left(t-t^{\prime}\right) \approx(\gamma / 2) \delta\left(t-t^{\prime}\right)$, where $\gamma=\omega_{A}^{3} d_{21}^{2} / 3 \pi \epsilon_{0} \hbar c^{3}$ is the usual decay rate for spontaneous emission in the absence of the PBG materials. However, the density of states of the photonic crystals exhibits band edge and other Van Hove singularities as described in the introduction. In such a system with fast variations of the density of states in the spectral range given by $\left\{\omega_{L}-2 \Omega, \omega_{L}+2 \Omega\right\}$ (shown in Figure 4), the distinctive memory functions introduced previously lead to qualitatively different behavior from ordinary vacuum. The description of the atomic evolution is further simplified by making the secular approximation i.e., the fast oscillating terms with frequencies $2 \Omega$ and $4 \Omega$ are discarded.

For weak and moderate external fields, the Mollow's spectral components may remain close to the density of states discontinuity and it is necessary to solve equations of motion generated by the Hamiltonian Eq. (3) without any additional approximation such as the Markov approximation.

In ordinary vacuum, the atomic system does not exhibit steady state inversion. Consequently, conventional lasers require additional atomic levels to achieve atomic inversion. In a PBG material, for a given intensity of the laser field, the atomic system reaches positive inversion (see Figure 5), and if the jump in the photonic density of states is quite large, the atomic system achieves nearly total inversion. This behavior is a consequence of the fact that the dressed state $|\tilde{1}\rangle$ (the left Mollow's sideband at the frequency $\omega_{L}-2 \Omega$ ) is placed in the spectral region with a low density of states and with slow decay, whereas the dressed state $|\tilde{2}\rangle$ (the right Mollow's side band at the frequency $\omega_{L}+2 \Omega$ ) experiences a large density of states and a rapid decay. In the long time limit, the population on the dressed state $|\tilde{1}\rangle$ is much larger than the atomic population in the dressed state $|\tilde{2}\rangle$. This imbalance of the atomic population between the dressed states is responsible for the atomic inversion in the bare picture.

Consider a near resonant laser excitation $\left(\omega_{L} \approx \omega_{A}\right)$. If we begin with an atom with resonant transition frequency, $\omega_{A}$, just outside the photonic band gap (say, for instance, $\Delta_{A C} \equiv \omega_{A}-\omega_{C}>0$ ), it is possible to drive the system through the transition described above, simply by increasing the applied field intensity. For a nearly resonant laser excitation $\left(\omega_{L} \approx \omega_{A}\right)$, the left Mollow side-band (at frequency $\omega_{A}-2 \Omega$ ) passes through the photonic band edge frequency when $\Omega_{c r i t}=\Delta_{L C} / 2$, while the other Mollow spectral components remain outside the gap. It is apparent from Figure 5 that for moderate values of the laser intensity, the atomic system switches very sharply from the ground state to the excited state, at a critical value of $\epsilon$. This switching behavior 


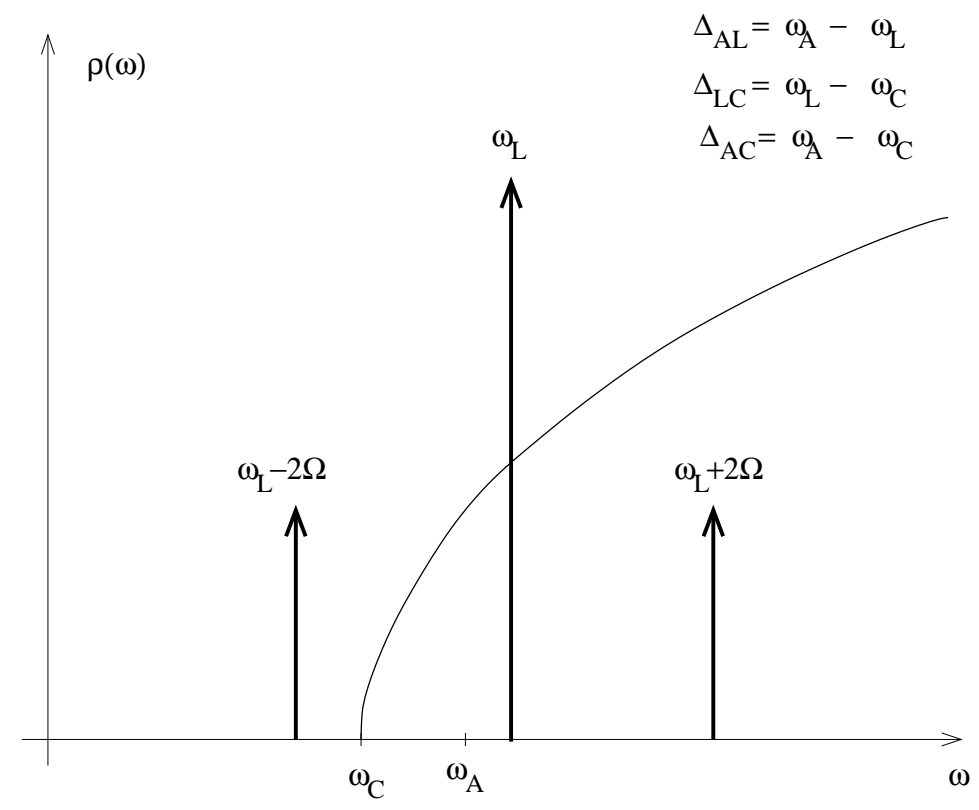

Figure 4. Relevant frequencies and frequency scales in the case of an anisotropic density of states. The laser frequency $\omega_{L}$, and the atomic frequency, $\omega_{A}$, are slightly positively detuned from the band edge frequency $\omega_{C}$.

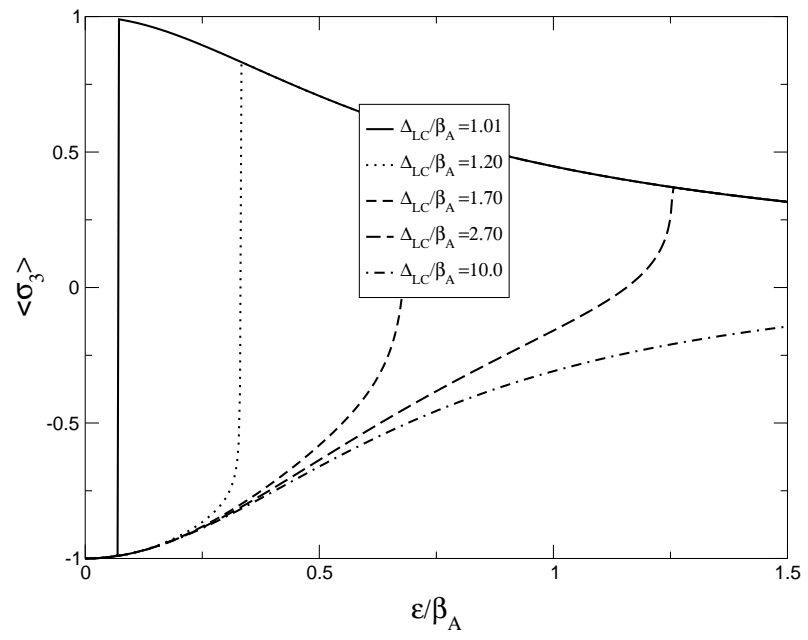

Figure 5. Atomic population inversion, $\left\langle\sigma_{3}\right\rangle$, as a function of the laser intensity $\epsilon / \beta_{A}$ for different values of the laser frequency detuning: $\Delta_{L C} / \beta_{A}=1.01$ (solid curve), $\Delta_{L C} / \beta_{A}=1.20$ (dotted curve), $\Delta_{L C} / \beta_{A}=1.70$ (dashed curve), $\Delta_{L C} / \beta_{A}=2.70$ (long-dashed curve), $\Delta_{L C} / \beta_{A}=10.0$ (dot-dashed curve). The atomic detuning is $\Delta_{A L}=-\beta_{A}$. 
is caused by the very sensitive dependence of the dressed atomic population on the relative position of the Mollow spectrum components. The magnitude of the effect depends on the actual value of the laser detuning with respect to the band edge frequency $\Delta_{L C}=\omega_{L}-\omega_{C}$.

\section{GAIN SPECTRUM FOR A WEAK PROBE BEAM}

In this section we analyze the gain spectrum of second laser field which probes the driven atomic system. For simplicity of illustration, consider a single atom, driven by an external laser field, and placed in a photonic crystal, exhibiting a step-like discontinuity in the density of states (the one atom version of the system analyzed in previous section). This simplified picture allows us to obtain analytical results and to provide a very intuitive picture of the switching and transistor action mechanisms in photonic crystals. It also provides a lower bound for the nonlinear switching effects. There are be two major enhancements to this worst case, the first coming from the non-Markovian character of a true band edge discontinuity (leading to lower threshold single atom switching as presented in Section 3), and the second one coming from the collective response of a system a two-level atoms placed in a photonic crystal (leading to larger differential gain and faster switching as suggested in Refs. 4,9). These additional effects provide a dramatic increase of the sensitivity, magnitude, and speed of the switching and transistor effects.

\subsection{Absorption and Dispersion of a Weak Probe Beam}

We now consider that in addition to the pump laser field, the atomic system is probed by a second laser field, of frequency $\omega \cdot{ }^{26-28}$ The probe field is assumed to be sufficiently weak to not disturb the dressed picture of the atomic system. The linear susceptibility of this system is given in terms of an electric field correlation function evaluated in the absence of the probe field ${ }^{4,25}$

$$
\chi(\omega)=i \mathcal{A} \int_{0}^{\infty}\left\langle\left[\mathcal{E}_{+}(t), \mathcal{E}_{-}(0)\right]\right\rangle_{s t} e^{i \omega t} d t,
$$

Here, $\mathcal{A}$ is a normalization constant, the index $s$ indicates that the average is evaluated in the steady state limit, and $\mathcal{E}_{ \pm}$are the positive and negative frequency components of the scattered electric field. In the ordinary vacuum case $\left(\gamma_{-}=\gamma_{+}=\gamma_{0}\right)$, the positive and negative frequency components of the scattered electric field are taken to be proportional with the atomic dipole operator

$$
\sigma_{12}(t)=\quad c s R_{3} e^{-i \omega_{L} t}-s^{2} R_{12} e^{-i\left(\omega_{L}-2 \Omega\right) t}+c^{2} R_{21} e^{-i\left(\omega_{L}+2 \Omega\right) t}
$$

However, in the case of a photonic crystal, the atomic dipole moment has spectral components that encounter different photonic density of states, and, implicitly, have different radiative rates $\left(\gamma_{0}, \gamma_{-}, \gamma_{+}\right)$. The positive and negative frequency components of the scattered electric field are given by ${ }^{4,29}$

$$
\begin{array}{ll}
\mathcal{E}^{+}(t)= & \sqrt{\frac{\gamma_{0}}{2}} \operatorname{cs} R_{3}(t)+\sqrt{\frac{\gamma_{+}}{2}} c^{2} R_{12}(t) e^{-2 i \Omega t}-\sqrt{\frac{\gamma_{+}}{2}} s^{2} R_{21}(t) e^{2 i \Omega t} \\
\mathcal{E}^{-}(t)= & {\left[\mathcal{E}^{-}(t)\right]^{\dagger} .}
\end{array}
$$

Using Eq. (12) and the fact the off-diagonal elements of the density matrix operator vanish in the stationary limit, in the strong driving field limit the central component at the frequency $\omega_{L}$ disappears, and the linear susceptibility has two well separated components, at the frequencies $\omega_{L} \pm 2 \Omega^{25,30}$

$$
\chi(\omega)=\chi^{(+)}(\omega)+\chi^{(-)}(\omega),
$$

with

$$
\begin{aligned}
\chi^{(+)}(\omega) & =i \mathcal{A} \gamma_{+} c^{4} \int_{0}^{\infty}\left\langle\left[R_{12}(t), R_{21}\right]\right\rangle_{s t} e^{i\left[\omega-\left(\omega_{L}+2 \Omega\right)\right] t} d t \\
\chi^{(-)}(\omega) & =i \mathcal{A} \gamma_{-} s^{4} \int_{0}^{\infty}\left\langle\left[R_{21}(t), R_{12}\right]_{s}\right\rangle e^{i\left[\omega-\left(\omega_{L}-2 \Omega\right)\right] t} d t
\end{aligned}
$$






Figure 6. The real and imaginary part of the linear susceptibility of the probe beam as a function of the detuning of the probe beam frequency, $\omega$, with respect to the driving laser field frequency $\omega_{L}$, for $\epsilon /\left|\Delta_{A L}\right|=0.178, \gamma_{-} / \gamma_{+}=0.001$, $\gamma_{0} / \gamma_{+}=1, \epsilon / \gamma_{+}=2.5$ and in the presence of a small amount of phonon mediated dephasing, $\gamma_{p} / \gamma_{+}=0.1$. The atomic resonant frequency is detuned negatively from the laser field frequency, $\Delta_{A L}=\omega_{A}-\omega_{L}<0$.

The correlation function present in Eq. (15) can be evaluated using the quantum regression theorem ${ }^{31}$ and the the real and imaginary parts of components of the linear susceptibility $\chi(\omega)$ are presented in Figure 6.

Similar to the ordinary vacuum case, ${ }^{26}$ the imaginary part of the linear susceptibility consists in one absorptive component and one amplifying component. In contrast to ordinary vacuum, the photonic crystal allows us to control the absorptive or amplifying character of the individual components (through variations in the intensity of the driving laser field). In particular, it is possible to switch a given sideband from being absorptive to exhibiting gain through a small change in the pump laser intensity. Unlike the fluorescence spectrum, absorption signal is proportional to the difference of the population between the dressed levels involved in the transition (multiplied by the transition rate between the levels). In contrast, the fluorescent signal depends only on the population of the initial state of the transition (multiplied by the transition rate). ${ }^{25,30}$ Clearly, the character of the absorption signal components is determined by the sign of the dressed atomic inversion, which in turn is determined by the intensity of the driving field (see Figure 7). In Figure 6, we plot the real and imaginary part of the linear susceptibility of the probe beam as a function of the detuning of the probe beam frequency, $\omega$, with respect to the driving laser field frequency $\omega_{L}$.

In ordinary vacuum, one Mollow sideband exhibits weak gain and the other sideband exhibits strong absorption. However, there is no switching of a given sideband with pump intensity. In the photonic crystal switching takes place. Moreover, the amplitude of the amplifying component is of the same order magnitude as the the amplitude of the absorbing component and easily detectable in a pump-probe spectroscopy experiment. The components are separated by a spectral distance proportional to the intensity of the driving field and their relative magnitude can be optimized by variations in the driving field. As the intensity of the driving laser field passes through the threshold intensity, the active medium switches from an absorptive medium to a gain medium at a relatively well defined intensity of the driving field.

\section{CONCLUSIONS}

We have analyzed the atomic switching in photonic crystals and suggested its relevance to all-optical transistor action. As a function of the intensity of the pump (control) laser, the active region of a photonic material (the atomic system) sharply switches from an absorptive medium (the atom spends most of its time in the ground state) to a gain medium (higher probability to find the atom in its excited state). This is a very fundamental effect arising from the modification of the electromagnetic vacuum in a photonic crystal. This 




Figure 7. The imaginary part of the linear susceptibility of the probe beam (absorption spectrum) as a function of the detuning of the probe beam frequency, $\omega$, with respect to the driving laser field frequency $\omega_{L}$, for $\gamma_{-} / \gamma_{+}=0.001$, $\gamma_{0} / \gamma_{+}=1, \epsilon / \gamma_{+}=2.5$ and in the presence of a small amount of phonon mediated dephasing, $\gamma_{p} / \gamma_{+}=0.1$. The two curves correspond to different Rabi frequencies, $\epsilon /\left|\Delta_{A L}\right|$, one of them (continuous curve) below the inversion threshold $\epsilon /\left|\Delta_{A L}\right|=0.178$, and the other one (dashed curve) above the threshold intensity $\epsilon /\left|\Delta_{A L}\right|=0.189$. The atomic resonant frequency is detuned negatively from the laser field frequency, $\Delta_{A L}=\omega_{A}-\omega_{L}<0$, and for this choice of parameters, the threshold intensity is $\epsilon_{t h r} /\left|\Delta_{A L}\right|=0.183$.

effect is forbidden in ordinary vacuum by the Einstein rate equations. We have shown that a weak second probe laser beam experience a substantial differential gain when the pump (control) laser intensity is in the neighborhood of the threshold value. This switching of the response of a probe beam (at a given frequency) to a small change in the pump (control) beam is absent in ordinary vacuum. In the first model, we considered an atom in a PBG material coupled to a 3D photonic band edge and driven by an external laser field of moderate intensity. Using a non-Markovian analysis of the system, we demonstrated switching behavior as a function of both laser field intensity and detuning frequency. The condition that triggers this jump in the atomic population inversion, corresponds to driving the left component of the atomic spectrum inside the gap, while the central resonance and the right side band remain outside the gap.

Based on these results, we suggest that if both non-Markovian and collective enhancement ${ }^{4}$ are incorporated in a single model, many desirable features of all-optical switching and all-optical transistor action will follow. These include low-pumping threshold, very large differential optical gain, and very fast response time, all in a very compact (20 micron scale) device. In addition to steady state operation as an all-optical transistor, it is of considerable interest to modulate the pump (control) field with input from an optical communication network. In this case, picosecond pulses of light could modulate the total pump (control) field back and forth across the switching threshold. In this way, pulses of light from the probe beam could be routed (amplified rather than absorbed) through the device, depending on whether a pulse from the pump field has reached the device. This provides an all-optical packet switching function. Since the gain spectrum experienced by the probe beam can be controllably displaced in frequency from the (modulated) pump frequency, this system could be used as an all-optical wavelength converter for data, from the pump to the probe. The relatively narrow spectral range of amplification of the proposed device can be dramatically increased by using an inhomogeneous active medium, with a broad distribution of resonant atomic transition frequencies. This occurs quite naturally if the active layers consists of quantum dots exhibiting a distribution of sizes. In this case the gain spectrum (and corresponding bandwidth of the switching device) may be considerably broadened relative to the narrow fluorescence side band shown in Figure 7. A second type of inhomogeneous broadening occurs due to local environments experienced by individual quantum dots. In particular, the local electromagnetic density of states will vary from point to point in space, leading to broadening of the spectral region available for switching. It is of 
considerable interest in this context to evaluate local density of states (LDOS) discontinuities for realistic device heterostructures and to examine in detail the relationship between the switching threshold energies, switching speeds, and corresponding coupling speeds of optical information in and out of the device.

A physical realization of this all-optical switching system could come from the growth of a III-IV semiconductor device heterostructure around a suitable PBG template. The heterostructure would consists of an active region of quantum dots sandwiched within a planar waveguide with an engineered electromagnetic DOS, between semiconductor cladding layers above and below exhibiting a large 3D PBG. The quantum dots would then "feel" the electromagnetic DOS presented by the defect layer within the larger PBG. Due to the size distribution of quantum dots, there would be a natural broadening of the gain spectrum experienced by the probe beam as discussed above. Positional randomness of the dots within the semiconductor backbone would also lead to "inhomogeneous" broadening of the switching threshold. An alternative realization of the effects described in this paper is through trapping and cooling real atoms in the void regions of a PBG crystal. The electric field distribution for a laser mode in the vicinity of the upper band edge (the so called "air" band) has strong intensity peaks in the void region of the material, which can act as an optical trap for active atoms. ${ }^{24,32}$ The trapped atoms will exhibit little interaction with the lattice of the dielectric host, thus minimizing additional decay and dephasing effect. A third possible realization of the all-optical switching we discussed, is from real atoms embedded in the dielectric backbone of a PBG material. The radiative transition of the erbium atom at comes from the atomic $4 f$ shell, which is screened by the outer shells from the environmental influence. At low temperatures, the erbium atoms suitably implanted in a silicon based PBG material may have very sharp single atom-like features. ${ }^{33}$

The central question in the realization of a PBG all-optical transistor is whether dielectric microstructure exhibiting a sufficiently large jump in the local electromagnetic density of states over a sufficiently small frequency interval can be realized in practice. While an ideal band edge exhibits a large jump, it is well known that the density of states associated with a real band edge can differ considerably from that of mathematical idealization when disorder effects and finite sample effects are considered. Another option is to engineer a more specific density of states profile by selectively introducing defect bands inside the large 3D PBG of the host photonic crystal. This may offer more flexibility in designing the optimum device heterostructure.

\section{REFERENCES}

1. H.M. Gibbs, Optical Bistability: Controlling Light with Light, Academic Press Inc., New York, 1985.

2. D.A.B Miller in Optical Computing, eds. B.S. Wherrett and F.A.P. Tooley, Proceedings of the 34th Scottish Universities Summer School in Physics, Edinburgh, August 1988 (Bristol: Adam Hilger), 1989.

3. H.M. Gibbs, et. al. Phys. Rev. Lett 36 1135, 1976.

4. S. John and M. Florescu, J. Opt. A. 3, S103, 2001.

5. S. John, Phys. Rev. Lett $\mathbf{5 3}$ 2169, 1984.

6. S. John, Phys. Rev. Lett $\mathbf{5 8}$ 2486, 1987.

7. E. Yablonovitch, Phys. Rev. Lett 58 2059, 1987.

8. S. John and T. Quang, Phys. Rev. A 50, 1764, 1994; A. Kaufman, G. Kurizki and B. Sherman, J. Mod. Opt. 41 353, 1984; M. Lewenstein, Zakrzewski J, and T.W. Mossberg, Phys. Rev. A 38 808, 1998.

9. S. John and T. Quang, Phys. Rev. Lett 78 1888, 1997.

10. R. Loudon, The quantum theory of light Oxford University Press, Oxford, 1995.

11. M. Florescu and S. John, Phys. Rev. A $64033801,2001$.

12. E. Yablonovitch, T.J. Gmitter and K.M. Leung, Phys. Rev. Lett 67 2295, 1991.

13. S.Y. Lin et. al., Nature 394 251, 1998.

14. K.M. Ho et. al., Solid State Com. 89 413, 1991.

15. A. Birner et. al., Adv. Mater. 13 377, 2001.

16. M. Cristophersen et. al., Mater. Sci. Eng. B 69 194, 2000.

17. R. Klose and K. Dichtel, unpublished.

18. W.L. Wos,Phys. Rev. B53 16231, 1996. 
19. J.E.G.L. Winjnhoven and W.L. Vos, Science $\mathbf{2 8 1} 802,1998$.

20. H. Miguez et. al., Phys. Rev. B59 1563, 1999.

21. Y.A. Vlasov, N. Yao and D.J. Norris Adv. Mater. 11 165, 1999.

22. A. Blanco et. al., Nature 405 437, 2001.

23. O. Toader and S. John, Science 229 5519, 2001.

24. O. Toader, S. John and K. Busch, Optics Express 8 272, 2001.

25. C. Cohen-Tannoudji, J. Dupont-Roc and C. Grynberg, Atom-Photon Interactions Wiley-Interscience, New York, 1992; B.R. Mollow, Phys. Rev. A 5 2217, 1972; H. Freedhoff and T. Quang, J. Opt. Soc. Am. B 12 474, 1994.

26. B.R. Mollow, Phys. Rev. A 5 2217, 1972.

27. E. Erhard and C.H. Keitel, Opt. Commun. 179 517, 2000.

28. O. Kocharovskaya and Y.Y. Radeonychev, Quantum Semiclass. Opt. 7 17, 1996.

29. T.W. Mosseberg and M. Lewenstein, J. Opt. Soc. Am. B 10 340, 1993.

30. C. Cohen-Tannoudji and S. Reynaud in Multiphoton Processes, J.H. Eberly, P. Lambropoulos, eds., Proceedings of an International Conference at the University of Rochester, June 1977, John Wiley \& Sons, New York, 1978.

31. M. Lax, Phys. Rev. 172 350, 1968.

32. M. Woldeyohannes and S. John, Phys. Rev. A 60 5046, 1999.

33. S. Lanzerstorter et. al., Appl. Phys. Lett. 72 809, 1998; V. F. Masterov et. al. , Appl. Phys. Lett. 72728 , 1998. 